\title{
Anchoring of Organic Molecules to a Metal Surface: HtBDC on Cu(110)
}

\author{
M. Schunack, ${ }^{1}$ L. Petersen, ${ }^{1}$ A. Kühnle, ${ }^{1}$ E. Lægsgaard, ${ }^{1}$ I. Stensgaard, ${ }^{1}$ I. Johannsen, ${ }^{2}$ and F. Besenbacher ${ }^{1} *$ \\ ${ }^{1}$ CAMP and Institute of Physics and Astronomy, University of Aarhus, DK-8000 Aarhus C, Denmark \\ ${ }^{2}$ Ris $\phi$ National Laboratory, Condensed Matter Physics and Chemistry Department, DK-4000 Roskilde, Denmark
}

(Received 1 September 2000)

\begin{abstract}
The interaction of largish molecules with metal surfaces has been studied by combining the imaging and manipulation capabilities of the scanning tunneling microscope (STM). At the atomic scale, the STM results directly reveal that the adsorption of a largish organic molecule can induce a restructuring of a metal surface underneath. This restructuring anchors the molecules on the substrate and is the driving force for a self-assembly process of the molecules into characteristic molecular double rows.
\end{abstract}

DOI: $10.1103 /$ PhysRevLett.86.456

Self-assembly of molecules on surfaces plays a vital role in the rapidly growing area of nanotechnology, especially within areas such as molecular electronics, nanodevices, and molecular recognition [1]. The supramolecular aggregation is most often controlled by noncovalent moleculemolecule interactions, and the metal surface is in most cases considered a static checkerboard that provides bonds and specific adsorption sites to the molecules [2].

However, when the adsorbed molecules become large and complex, the complexity of the interaction between the substrate and the molecules may increase. In previous studies different groups have suggested that the formation of self-assembled monolayers is in general controlled by a subtle balance between the competing intermolecular and molecule-substrate interactions. In a few cases it has been indicated, mainly from measured height corrugations of the adsorbed molecules, that a restructuring of the substrate may exist underneath the molecular adsorbate layer $[3,4]$. However, no firm conclusions have been reached and in some cases conflicting evidence has been published [5].

In this Letter we report scanning tunneling microscopy (STM) results, which directly demonstrate that anchoring of largish molecules and the subsequent self-assembly of molecular nanostructures on a metal surface can indeed be associated with a local disruption of the surface layer underneath the molecules. Identifying the exact nature of such a restructuring may be difficult when using an averaging surface sensitive probe such as standard scattering techniques. However, by combining the ultimate resolution imaging capabilities of the STM with its ability to manipulate single molecules in a controlled manner [6], we have at the atomic scale directly revealed that, associated with the adsorption of largish molecules, metal atoms can be dug out of the surface, resulting in a "trench base" for anchoring of the molecules. These results prove that molecule-surface interactions can be a controlling driving force for self-assembly of molecules on surfaces.

The molecule-surface system under investigation is hexa-tert-butyl-decacyclene molecules $\left(\mathrm{HtBDC}, \mathrm{C}_{60} \mathrm{H}_{66}\right)$ on a $\mathrm{Cu}(110)$ surface [7]. The largish HtBDC molecule is depicted in Fig. 1 and possesses the basic structural features required for future molecular building blocks
PACS numbers: 68.35.Bs, 68.37.Ef, 61.46.+w, 68.43.-h

for electronic devices: a conducting backbone (aromatic $\pi$ system) and spacer groups ( $t$-butyl groups, $-\mathrm{C}_{4} \mathrm{H}_{9}$ ), separating the conducting parts of the molecule from the substrate.

The experiments were performed in an ultrahigh vacuum chamber equipped with a very stable, homebuilt, low and variable-temperature STM, capable of operating at 25-400 K [8]. The $\mathrm{Cu}(110)$ surface was sputter cleaned by $2.0 \mathrm{keV} \mathrm{Ne}$ ion bombardment followed by annealing at $820 \mathrm{~K}$. The HtBDC powder was transferred onto the $\mathrm{Cu}(110)$ surface by organic molecular beam deposition out of a resistively heated glass crucible at $450 \mathrm{~K}$, while the substrate was kept at room temperature (RT).

At very low coverages the molecules are observed to decorate the steps, indicating that the diffusion barrier for the individual molecule on the flat $\mathrm{Cu}(110)$ surface is low enough to allow the molecules to be mobile. With increasing coverages the STM images reveal the formation of self-assembled double rows of HtBDC molecules aligned along the equivalent [1 12$]$ and [11 2$]$ directions (Fig. 2a). These characteristic double row structures appear to nucleate homogeneously over the terraces and are comprised of subunits consisting of two molecules with different orientation.

Imaging the same structure at low temperatures, $T<$ $160 \mathrm{~K}$, single, immobile molecules appear in addition to
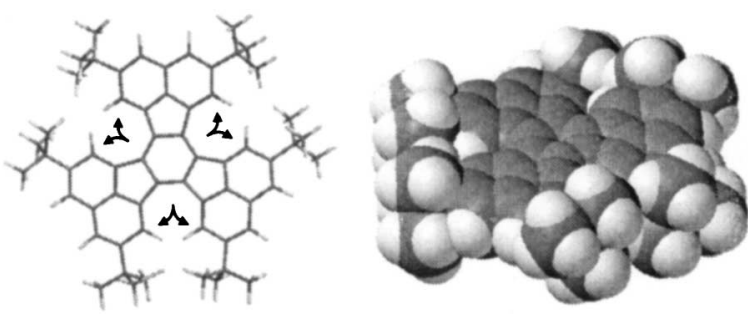

FIG. 1. Tube and space-filling model of HtBDC showing the propeller-shaped conformation of the molecule. HtBDC consists of an aromatic ring system (decacyclene) built from a centered benzene ring interconnected by three five-membered rings to three naphthalene subunits (each with two butyl groups attached to it). The pairs of $\mathrm{H}$ atoms responsible for the distortion from a planar molecule conformation are indicated by arrows. 


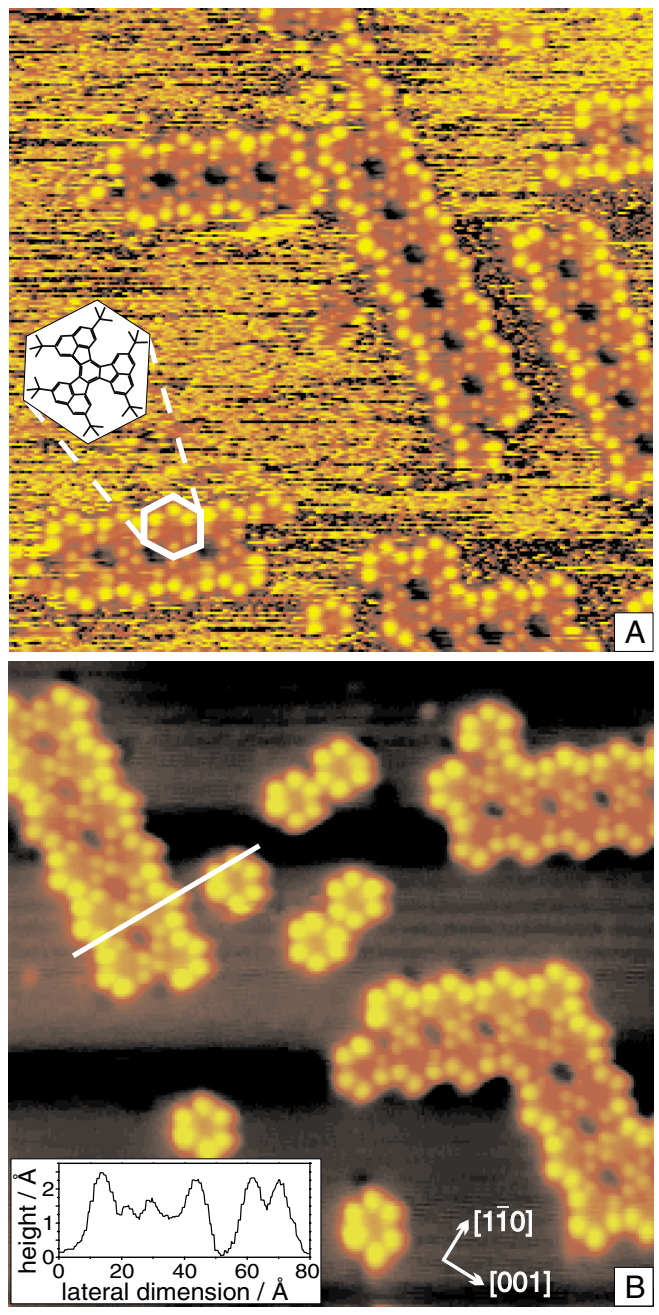

FIG. 2 (color). Constant current STM images of HtBDC on $\mathrm{Cu}(110)$ at low coverage. (a) At room temperature $\left(V=1051 \mathrm{mV}, I=0.43 \mathrm{nA}, 200 \times 200 \AA^{2}\right)$. The orientation of the six lobes in the imaged molecules is illustrated in the inserted model. (b) At $T \simeq 26 \mathrm{~K}(V=1250 \mathrm{mV}, I=0.42 \mathrm{nA}$, $200 \times 200 \AA^{2}$ ). The inset shows the height plot along the white line in $\AA$ units; the arrows indicate the crystal directions.

the double row structures. They show a planar geometry with the six lobes arranged in a distorted hexagon with threefold rotational symmetry (e.g., in Fig. $2 b$ at $26 \mathrm{~K}$ ), consistent with the disklike geometry of the molecules. According to the dimensions of the lobes and elastic scattering quantum chemistry calculations for $\mathrm{HtBDC}$ on $\mathrm{Cu}(100)$ [9], each lobe can be assigned to one of the $t$-butyl appendages. The two observed orientations of the single, individual molecules adsorbed on the flat surface correspond to two equivalent adsorption sites. Rotation of a molecule by $60^{\circ}$ (or $180^{\circ}$ or $300^{\circ}$ ) changes nothing with respect to the adsorption site, since the $\mathrm{Cu}(110)$ surface has a mirror plane perpendicular to the [11 10$]$ direction.

At higher temperatures, $T>160 \mathrm{~K}$, a one-dimensional diffusion of the single molecules along the [1iㅣㄹ direction is found to set in. From STM movies, i.e., series of time-lapsed STM images [10], the activation barrier for diffusion of the molecules, $E_{\mathrm{D}}=0.36 \mathrm{eV}$, has been determined from the hopping rates by tracking the positions of the individual molecule. Furthermore, at RT STM movies [11] show that the double row unit fluctuates in size, growing or shrinking at the ends, but the rows do not diffuse as a unit. On a time scale of seconds, new rows are observed to nucleate (containing at least two HtBDC molecules) and existing rows disappear. Thus, the streaks at RT in the intermediate areas between the self-assembled double rows of HtBDC molecules (Fig. 2a) are associated with single, individual HtBDC molecules diffusing faster than the time scale of STM imaging (5-30 s/image).

From Fig. $2 b$ it is evident that the single, individual molecules are imaged very differently compared to the molecules within the double rows. For the single molecules all six lobes appear to have nearly the same height relative to the $\mathrm{Cu}$ surface $(\simeq 2.2 \pm 0.1 \AA)$. For the molecules within a double row, however, the three lobes at the rim of the row are imaged much brighter than the three lobes pointing towards the interior of the double row (see line scan in Fig. 2b). The height difference scatters around $1 \AA$, close to the step height on a $\mathrm{Cu}(110)$ surface $(1.28 \AA)$. Thus, whereas single, isolated molecules are adsorbed with the molecule plane parallel to the $\mathrm{Cu}(110)$ surface, the molecules in the double rows appear to be tilted. A purely electronic reason for these imaging differences due to the proximity of neighboring molecules can be excluded. On one hand, adjacent molecules with six identical lobes each are present (Fig. 2b) and, on the other hand, manipulating two single, individual molecules "into contact" did not lead to any change in their appearance.

To explore the two different imaging modes of the HtBDC molecules, we have utilized the unique ability of the STM to manipulate single molecules on surfaces on the atomic scale [6]. The controlled manipulation of the molecules is performed by reducing the tunneling resistance from $1 \mathrm{G} \Omega$ to $1 \mathrm{M} \Omega$ by changing the tunneling current or voltage or both. This corresponds to a tip surface approach of around $3 \AA$. When scanning with this reduced tunneling resistance, all $\mathrm{HtBDC}$ molecules will be pushed outside the scanned area after two to four scans.

In Fig. 3 is shown an STM image recorded at $41 \mathrm{~K}$ of (a) an area with double rows of molecules before manipulation and (b) a "cleaned" surface area after the molecules are pushed aside. The latter STM image directly discloses the existence of a local disruption of the topmost $\mathrm{Cu}$ surface layer. We find that $\simeq 14 \mathrm{Cu}$ atoms are expelled from the surface in two adjacent [1 10$]$ rows, forming a trenchlike base for anchoring of the molecules. From atomically resolved images where the molecular double rows and the $\mathrm{Cu}(110)$ lattice are resolved simultaneously, the registry of the molecules can be determined. The three more dimly imaged $t$-butyl lobes of each molecule are located on top of the missing $\mathrm{Cu}$ atoms. In Fig. $3 \mathrm{c}$ is shown a schematic ball model of this novel structure, and, as can be seen, the 

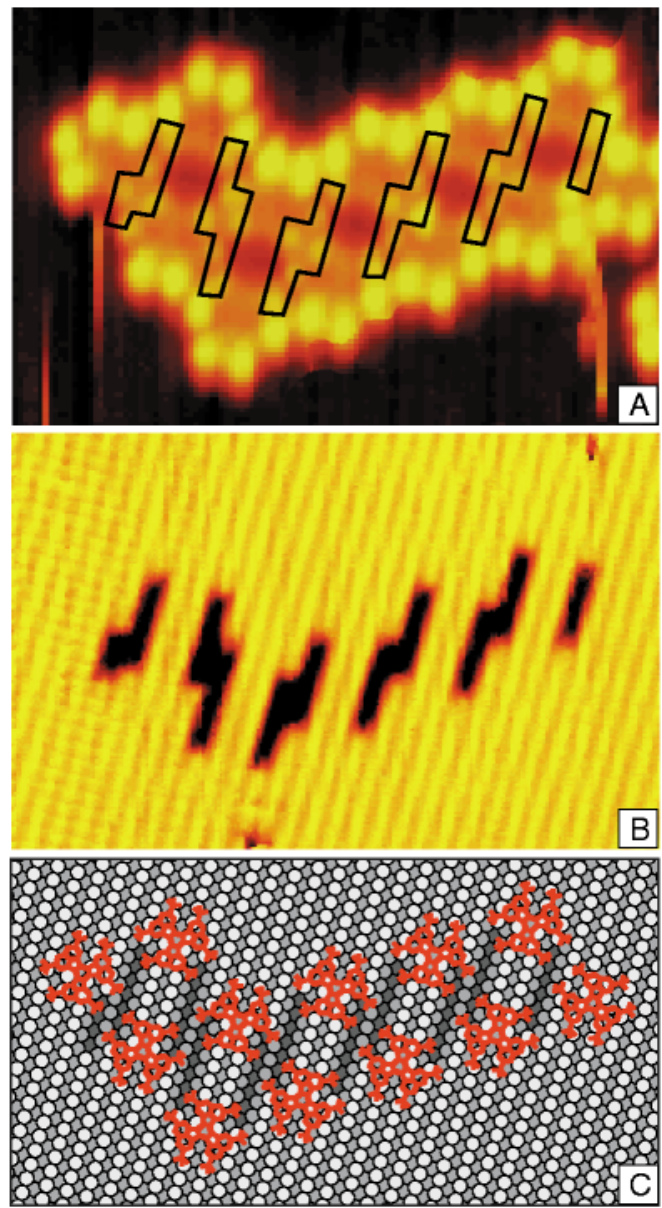

FIG. 3 (color). Constant current images $\left(105 \times 69 \AA^{2}\right)$ at $41 \mathrm{~K}$. (a) HtBDC double row structure $(V=1070 \mathrm{mV}$, $I=0.45 \mathrm{nA})$. The trenches in the underlying surface are sketched. (b) The trenches in the surface layers are disclosed after manipulating the molecules aside $(V=7 \mathrm{mV}$, $I=1.82 \mathrm{nA}$ ). Atomic resolution along the close-packed direction was obtained in the left part of the image (vertical fast scanning direction), whereas it was lost when the tip scanned the restructured area. (c) Ball model of the double row structure - the substrate atoms are shaded darker the deeper the layers lie, while the molecules are shown in red.

$t$-butyl groups of the HtBDC molecule inside the double rows lie in the trench, whereas the outer $t$-butyl groups lie on the plain $\mathrm{Cu}(110)$ surface (see also Fig. 3a).

What is the origin of the restructuring, i.e., the formation of the trenches in the surface layer? In general, the binding of adsorbates will induce a restructuring of the surface only if the gain in the adsorption energy $\Delta E_{\text {ad }}$ of the molecules on the disrupted surface, as compared to the virgin surface, is sufficiently high to balance the energy cost $\Delta E_{\text {metal }}$ required to break the metal bonds, i.e., the energy difference between the disrupted and the virgin clean surface.

The anchoring of molecules on the disrupted surface may have two reasons. On the one hand, it may be a simple steric effect, an adaptation of the surface geometry to allow the $t$-butyl groups to fit into the trenches, resulting in a larger interaction area. Alternatively, within the $d$-band model by Hammer and Nørskov [12], the creation of steps and even kink sites underneath the molecules causes a higher reactivity of the substrate and therefore a stronger binding, since a simple correlation exists between the bonding strength of a molecule and the metal coordination number of the adsorption site. Finally, it may also be a combination of the described effects.

A lower bound for $\Delta E_{\mathrm{ad}}$ of the HtBDC molecules can be estimated from $\Delta E_{\text {metal }}$ involved in removing the $\mathrm{Cu}$ atoms out of the surface, forming the trenches. Within the approximative effective medium theory (EMT) approach $[13,14]$, the energy $E_{i}$ of an atom in an fcc structure is a simple function of the nearest-neighbor $(\mathrm{nn})$ and nextnearest-neighbor (nnn) coordination numbers, $\left(N_{1}\right.$ and $N_{2}$, respectively), for the $\mathrm{Cu}$ atoms in the topmost surface layers:

$$
\begin{aligned}
E_{i}\left(N_{1}, N_{2}\right) \simeq & V_{2}\left(12-N_{1}\right) \\
& +\frac{1}{2} V_{3}\left(12-N_{1}\right)^{2}+V_{2}^{\prime}\left(6-N_{2}\right) .
\end{aligned}
$$

Using the parameters $V_{2}=0.109 \mathrm{eV}$ and $V_{2}^{\prime}=$ $0.0246 \mathrm{eV}$ for the effective $\mathrm{nn}$ and nnn pair interactions, respectively, and $V_{3}=0.0023 \mathrm{eV}$ for the effective $\mathrm{nn}$ three-body interaction for $\mathrm{Cu}$ [13], we can calculate the energies $E_{i}$ for every atom that changes its coordination number when being expelled from the close-packed $\mathrm{Cu}$ rows. In Fig. 4 and Table I we indicate a labeling of the atoms in the trench structure and the energies involved in forming the trench. Moving the first $\mathrm{Cu}$ atom "1" from the surface to an adatom site "a" is energetically the most expensive move $(0.56 \mathrm{eV})$; it involves the breaking of two nn bonds [creating (100) facets]. Extracting the next six atoms along the close-packed rows requires only $0.32 \mathrm{eV}$ each, since only one nn bond is broken per atom [creating (111) facets]. The expelling of the atoms from the adjacent close-packed $\mathrm{Cu}$ row ("8"-"14") resembles this behavior and differs only due to the number of nnn's. The overall energy used to remove the 14 atoms from the surface to adatom sites thus equals $4.83 \mathrm{eV}$. These adatoms can reduce their energy by diffusing to a kink site " $k$ " of a step, thereby each gaining $-0.28 \mathrm{eV}$ per atom, amounting in total to $-3.93 \mathrm{eV}$ for all 14 adatoms. The final energy required to form a trench is $\Delta E_{\text {metal }}=0.89 \mathrm{eV}$ [15], and the gain in adsorption energy per molecule by forming the trenches must thus be at least $0.45 \mathrm{eV}$, since (on average) two molecules share a single hole.

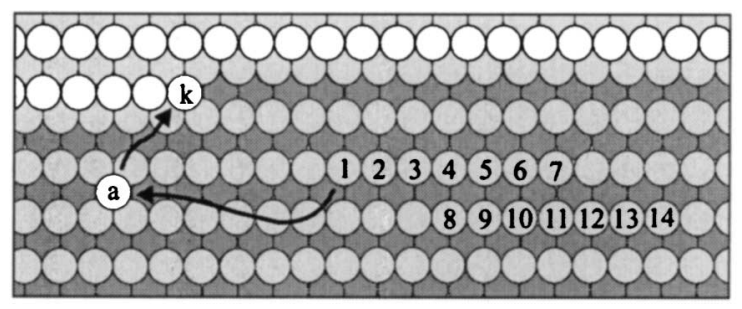

FIG. 4. Labeling of atoms referring to Table I. "a" marks an adatom, and "k" a kinkatom. 
TABLE I. Vacancy-adatom and adatom-kinkatom formation energies $\Delta E_{i}$ on $\mathrm{Cu}(110)$ in $\mathrm{eV}$ obtained successively corresponding to their label numbers (Fig. 4) by means of EMT.

\begin{tabular}{|c|c|c|c|c|c|}
\hline \multirow[b]{2}{*}{ Atom label $i$} & \multicolumn{4}{|c|}{ vac.-ad. } & \multirow{2}{*}{$\frac{\text { ad.-ki. }}{\mathrm{a} / \mathrm{k}}$} \\
\hline & 1 & $2-7$ & 8 & $9-14$ & \\
\hline$\Delta E_{i}$ & 0.56 & 0.32 & 0.52 & $\sim 0.3$ & -0.28 \\
\hline$\sum_{1}^{14} \Delta E_{i}$ & \multicolumn{4}{|c|}{4.83} & -3.93 \\
\hline
\end{tabular}

If the HtBDC molecules are deposited at sample temperatures below $\sim 250 \mathrm{~K}$, the molecular double row structures do not form, which is easily explained qualitatively within the above model. At these temperatures there is apparently not enough thermal energy available to promote the adatom-vacancy formation on the $\mathrm{Cu}$ substrate.

In conclusion our main new finding is that adsorption of largish molecules to metal surfaces and the subsequent formation of self-assembled monolayers can be associated with a disruption of the metal substrate, increasing the molecule-substrate interactions. The atomic-scale imaging and manipulation capability of the STM has been used to directly reveal such a restructuring process. HtBDC molecules are anchored to the $\mathrm{Cu}(110)$ surface through the formation of a characteristic trench base in which $14 \mathrm{Cu}$ atoms are dug out of the surface layer in two neighboring close-packed rows. The local nature of the moleculeinduced surface disruption implies that it would have been extremely difficult to identify it using other surface sensitive techniques. The spontaneous surface disruption formed underneath the largish molecules during the adsorption process is a generic way to reduce the mobility of the molecules and bind them to the surface at even low coverages. If the underlying forces and mechanisms of the observed adsorption processes are understood and can be used in a controlled manner by specially designed molecules, it also indicates a new route for nanostructuring of surfaces.

We acknowledge stimulating discussions with J.K. Nørskov, as well as the financial support from The Danish National Research Foundation through The Center for Atomic-Scale Materials Physics (CAMP), from the VELUX Foundation, and from the EU networks "Bottom up Nanomachines" and "Manipulation of individual atoms and molecules."

*Corresponding author.

Email address: fbe@ifa.au.dk

[1] G. M. Whitesides, J.P. Mathias, and C. T. Seto, Science 254, 1312 (1991); J. Seto et al., Thin Solid Films 273, 97 (1996); J.-P. Sauvage, Acc. Chem. Res. 31, 611 (1996); C. P. Collier et al., Science 285, 391 (1999); C. S. Lent,
Science 288, 1597 (2000); M. O. Lorenzo, C. J. Baddeley, C. Muryn, and R. Raval, Nature (London) 404, 376 (2000).

[2] J. V. Barth et al., Angew. Chem., Int. Ed. Engl. 39, 1230 (2000); A. K. Boal et al., Nature (London) 404, 746 (2000); O. Marchenko and J. Cousty, Phys. Rev. Lett. 84, 5363 (2000); M. Furukawa et al., Surf. Sci. 445, L58 (2000).

[3] For examples with $\mathrm{C}_{60}$ molecules, see E. I. Altman and R. J. Colton, Surf. Sci. 279, 49 (1992); J. K. Gimzewski, S. Modesti, Ch. Gerber, and R. R. Schlittler, Chem. Phys. Lett. 213, 401 (1993); A. J. Maxwell et al., Phys. Rev. B 52, R5546 (1995); P. W. Murray et al., Phys. Rev. B 55, 9360 (1997).

[4] For examples with thiols, amino acids, etc., see G.E. Poirier, J. Vac. Sci. Technol. B 14, 1453 (1996); X. Zhao, R. G. Zhao, and W. S. Yang, Surf. Sci. 442, L995 (1999); S. Haq and F. M. Leibsle, Surf. Sci. 355, L345 (1996); J. K. Gimzewski, T. A. Jung, M. T. Cuberes, and R. R. Schlittler, Surf. Sci. 386, 101 (1997); S. M. Driver and D. P. Woodruff, Langmuir 16, 6693 (2000).

[5] J. K. Gimzewski, S. Modesti, and R. R. Schlittler, Phys. Rev. Lett. 72, 1036 (1994); M. Pedio, R. Felici, X. Torrelles, P. Rudolf, M. Capozi, J. Rius, and S. Ferrer, Phys. Rev. Lett. 85, 1040 (2000).

[6] D. M. Eigler and E. K. Schweizer, Nature (London) 344, 524 (1990); L. Bartels, G. Meyer, and K.-H. Rieder, Appl. Phys. Lett. 71, 213 (1997); J. A. Stroscio and D. M. Eigler, Science 254, 1319 (1991); Ph. Avouris, Acc. Chem. Res. 28, 95 (1995).

[7] K. Zimmermann, R. Goddard, C. Krüger, and M. W. Haenel, Tetrahedron Lett. 37, 8371 (1996); D. M. Ho and R. A. Pascal, Jr., Chem. Mater. 5, 1358 (1993).

[8] L. Petersen, M. Schunack, B. Schaefer, T. R. Linderoth, P. B. Rasmussen, P.T. Sprunger, E. Lægsgaard, I. Stensgaard, and F. Besenbacher, Rev. Sci. Instrum. (to be published).

[9] J. K. Gimzewski, C. Joachim, R. R. Schlittler, V. Langlais, H. Tang, and I. Johannsen, Science 281, 531 (1998).

[10] S. Horch, H. T. Lorensen, S. Helveg, E. Lægsgaard, I. Stensgaard, K. W. Jacobsen, J. K. Nørskov, and F. Besenbacher, Nature (London) 398, 134 (1999).

[11] The movie is visualized on the World Wide Web: http://www.ifa.au.dk/camp/movies/htbdc.mpg

[12] B. Hammer and J. K. Nørskov, Nature (London) 376, 238 (1995); in Theory of Adsorption and Surface Reactions, Chemisorption and Reactivity on Supported Clusters and Thin Films, edited by R. M. Lambert and G. Pacchioni (Kluwer Academic Publishers, Amsterdam, 1997), pp. 285-351.

[13] O. B. Christensen and K. W. Jacobsen, Phys. Rev. B 45, 6893 (1992).

[14] J. K. Nørskov, K. W. Jacobsen, P. Stoltze, and L. B. Hansen, Surf. Sci. 283, 277 (1993).

[15] The main contribution to the energy of $0.89 \mathrm{eV}$ is from atoms " 1 " and "8." The difference in energies needed to start and to extend a row can be used to explain the packing of the molecules in double rows as a balance of van der Waals forces between the molecules and the formation energy of the vacancies, but it goes beyond the scope of this Letter. 